\title{
NOX2: a determinant of acute myeloid leukemia survival
}

Courtney L. Jones ${ }^{1,2}$

${ }^{1}$ Princess Margaret Cancer Centre, University Health Network, Toronto and ${ }^{2}$ Department of Medical Biophysics, University of Toronto, Toronto, Ontario, Canada

E-mail: courtney.jones@uhnresearch.ca

https://doi.org/10.3324/haematol.2022.280677

In this issue of Haematologica, Paolillo et al. investigate mechanisms of chemotherapy resistance in acute myeloid leukemia (AML). ${ }^{1}$ While $A M L$ therapy is evolving rapidly, many patients will receive conventional induction chemotherapy which consists of a 7-day continual infusion of cytarabine accompanied by infusions of an anthracycline, such as daunorubicin, on the first 3 days of treatment. Many patients receiving this therapy will respond but it is common for patients to develop disease recurrence and succumb to this disease. This is in part due to the development of resistance to chemotherapy. To understand the mechanistic underpinnings of therapy resistance in AML cells, Paolillo et al. generated cytarabine- and daunorubicin-resistant HL-60 cells and measured changes in gene expression. They discovered that NADPH oxidase 2 (NOX2) subunit expression was greatly elevated in daunorubicin-resistant cells and subunit $C Y B B$ was significantly increased in cytarabineresistant cells. Importantly, this correlated with an increase in NOX2 activity in daunorubicin-resistant cells. NOX2 has been well characterized in normal and malignant hematopoiesis. Indeed, NOX2 has been shown to be the most predominant oxidase expressed in human and murine AML. ${ }^{2}$ Interestingly, it is also highly expressed in hematopoietic stem cells and is functionally important for proper myelopoiesis. ${ }^{2}$ However, NOX2 had not previously been shown to promote chemotherapy resistance in AML cells.

Elevated NOX2 levels result in increased reactive oxygen species (ROS) which have been shown to promote AML cell proliferation during leukemia development. ${ }^{3,4}$ In contrast, no changes in proliferation were observed in daunorubicin-resistant lines compared to daunorubicinsensitive cells or upon knockout of the NOX2 subunit
CYBB in daunorubicin-resistant lines, demonstrating that NOX2-mediated daunorubicin resistance was not a result of a proliferative advantage. However, knockout of the NOX2 subunit CYBB did re-sensitize cells to daunorubicin, demonstrating that NOX2 was directly contributing to daunorubicin resistance in AML. Furthermore, treatment with a pan-NOX inhibitor restored sensitivity of daunorubicin-resistant cells to daunorubicin, indicating the potential for NOX2-targeted pharmacological interventions to restore chemotherapy sensitivity in therapy-resistant patients. However, as the authors note most NOX inhibitors lack specificity which raises toxicity concerns. In addition, reduction in NOX2derived ROS re-sensitized cells to daunorubicin showing that elevated ROS production was also an essential component of NOX2-mediated therapy resistance (Figure 1A). It is important to note that the relationship between ROS and cancer is very complex and an important area of tumor biology that continues to evolve. ROS can promote tumor formation and have been shown to be a potential therapeutic target. ${ }^{5}$ In AML, elevating ROS levels within the mitochondria can increase sensitivity to targeted AML therapies including FLT3 inhibitors. ${ }^{6}$ As eloquently described in a review by Harris and DeNicola, it is likely that these contradictory findings can be explained by nuanced differences in types of ROS, cellular localization of ROS, and the tissues being examined. ${ }^{5}$

Gene expression in AML can vary based on several factors, including mutational and differentiation status. To interrogate the potential heterogeneity of NOX2 expression in AML, Paolillo et al. quantified NOX2 subunit gp $91^{\text {phox }}$ protein expression in 74 AML specimens by flow cytometry and gene expression in a cohort from The Cancer Genome Atlas (TCGA). Notably, gp91 phox $^{\text {levels }}$
A

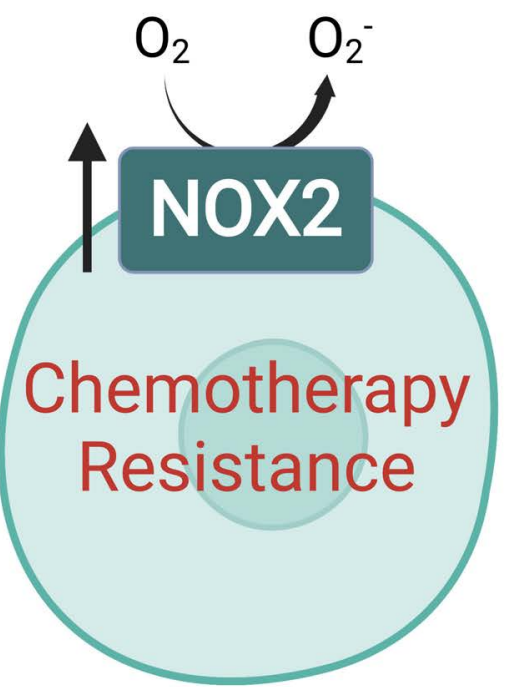

B

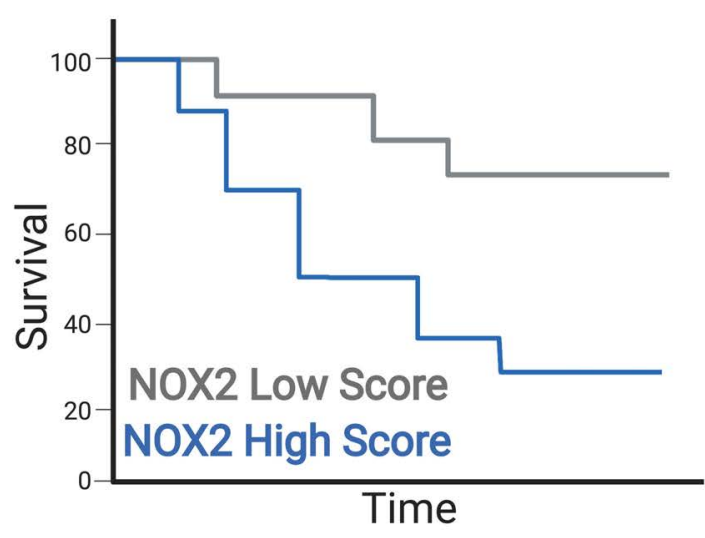

Figure 1. Graphical representation of the key findings of Paolillo et al. ${ }^{1}$ (A) Elevated levels of NADPH oxidase 2 (NOX2) promote chemotherapy resistance in acute myeloid leukemia (AML) through increased production of reactive oxygen species. (B) A high NOX2 score predicts poor outcomes for AML patients. Figure created with BioRender.com. 
correlated with the French, British, American (FAB) M4 and $\mathrm{M} 5$ classification compared to $\mathrm{M0}, \mathrm{M} 1$, and $\mathrm{M} 2$ at the protein and mRNA levels. In contrast, NOX2 subunit expression did not correlate with European LeukemiaNet status, or mutations in NPM1 or FLT3. These findings are particularly intriguing, as AML cases classified as M5 have been shown to exhibit increased resistance to the BCL-2 inhibitor venetoclax in combination with hypomethylating agents; ${ }^{7}$ however, NOX2 activity did not contribute to venetoclax resistance in these models. Furthermore, gp91 $1^{\text {phox }}$ levels and NOX2-derived ROS were higher in CD34- leukemic blasts compared to CD34+ leukemic stem cells (LSC). Importantly, LSC did display a basal level of NOX2 activity, consistent with NOX2 being essential for LSC function. ${ }^{2}$

Strikingly, Paolillo et al. demonstrated that a NOX2 gene expression score, which was developed by combining gene expression of each NOX2 subunit, was predictive of survival of AML patients (Figure 1B). Specifically, a higher NOX2 score correlated with decreased survival in three independent cohorts (Verhaak, Metzeler, and TCGA). Other gene expression scores containing NOX2 subunits have also been shown to be predictive of $A M L$ patient survival, ${ }^{8}$ highlighting the potential importance of NOX2 as a predictive biomarker in AML. Interestingly, the NOX2 score was higher for patients with a M4 or M5 FAB classification; however, the predictive value of the NOX2 score was independent of FAB classification. Therefore, the NOX2 score may have broad implications as a predictive biomarker for AML patients. Since NOX2 levels were higher in leukemic blasts than in LSC in the future it would be interesting to determine the overlap or potential combinatorial power of the NOX2 score with LSC-specific scores, such as the LSC17. ${ }^{9}$ Finally, in the past decade AML therapy has changed dramatically with the approval of therapies such as the BCL-2 inhibitor venetoclax, FLT3 inhibitor midostaurin, and IDH1 and IDH2 inhibitors ivosidenib and enasidenib. It will be particularly interesting and clinically important to determine whether gene expression signatures that predict resistance to chemotherapy, such as the NOX2 gene expression score, have prognostic value for other AML therapies.

Overall, Paolillo et al. defined a new mechanism of therapy resistance in AML, NOX2 overexpression. Mechanistically, NOX2 overexpression leads to elevated ROS levels which contribute directly to therapy resistance. Importantly NOX2 overexpression has prognostic value for AML patients treated with chemotherapy. This work provides the foundation for future studies aimed at determining the applicability of the NOX2 score as a predictive biomarker in the clinical setting.

\section{Disclosures}

No conflicts of interest to disclose.

\section{References}

1. Paolillo R, Boulanger M, Gâtel P, et al. The NADPH oxidase NOX2 is a marker of adverse prognosis involved in chemoresistance of acute myeloid leukemias. Haematologica. 2022;107(11):2562-2575.

2. Adane B, Ye H, Khan N, et al. The hematopoietic oxidase NOX2 regulates self-renewal of leukemic stem cells. Cell Rep. 2019;27(1):238-254.e6

3. Hole PS, Zabkiewicz J, Munje C, et al. Overproduction of NOXderived ROS in AML promotes proliferation and is associated with defective oxidative stress signaling. Blood. 2013;122(19):3322-3330.

4. Reddy MM, Fernandes MS, Salgia R, Levine RL, Griffin JD, Sattler M. NADPH oxidases regulate cell growth and migration in myeloid cells transformed by oncogenic tyrosine kinases. Leukemia. 2011;25(2):281-289.

5. Harris IS, DeNicola, GM. The complex interplay between antioxidants and ROS in cancer. Trends Cell Biol. 2020;30(6):440-451.

6. Gregory MA, D'Alessandro A, Alvarez-Calderon F, et al. ATM/G6PD-driven redox metabolism promotes FLT3 inhibitor resistance in acute myeloid leukemia. Proc Natl Acad Sci U S A. 2016;113(43):E6669-E6678.

7. Pei S, Pollyea DA, Gustafson A, et al. Monocytic subclones confer resistance to venetoclax-based therapy in patients with acute myeloid leukemia. Cancer Discov. 2020;10(4):536-551.

8. ljurko C, González-García N, Galindo-Villardón P, HernándezHernández Á. A 29-gene signature associated with NOX2 discriminates acute myeloid leukemia prognosis and survival. Am J Hematol. 2022;97(4):448-457.

9. Ng SW, Mitchell A, Kennedy JA, et al. A 17-gene stemness score for rapid determination of risk in acute leukaemia. Nature. 2016;540(7633):433-437. 